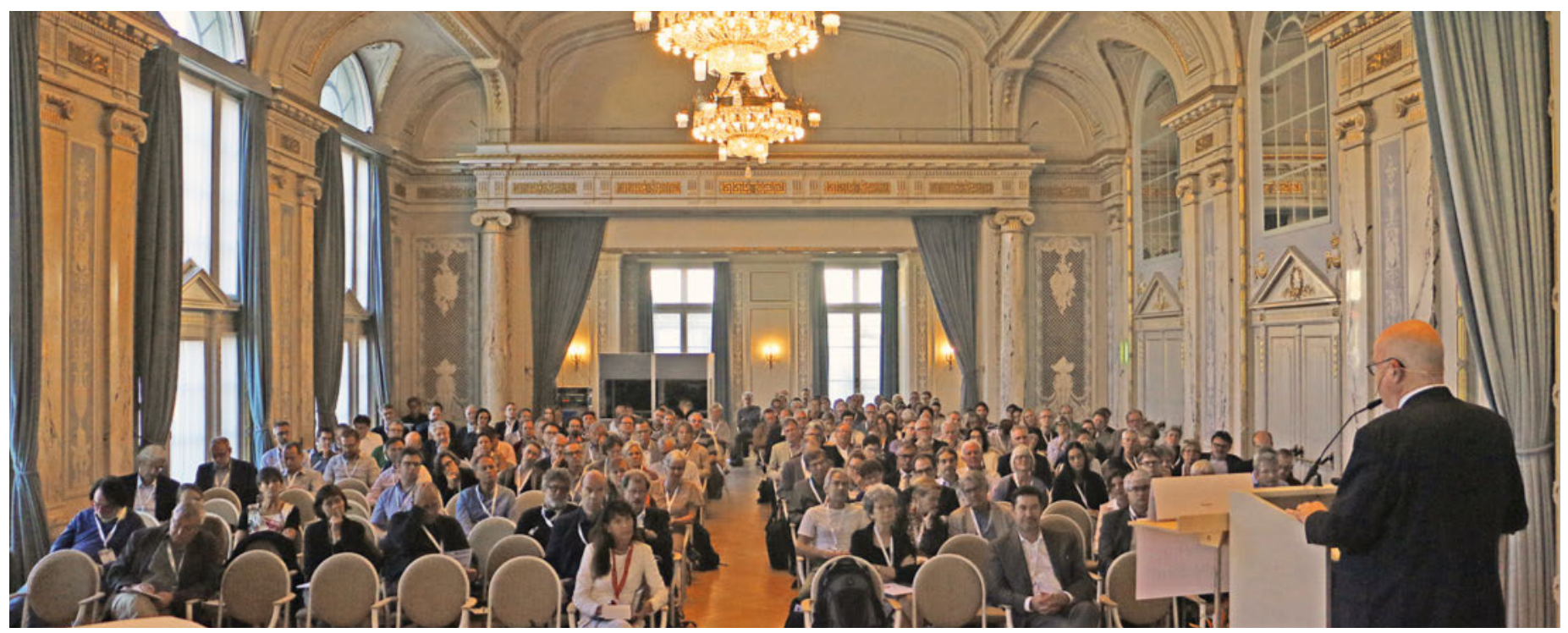

Auch die dritte Auflage des MedEd Symposiums stiess auf grosses Publikumsinteresse.

\title{
Ärztliche Weiterbildung: Sorgenkind mit Zukunft
}

\section{Bruno Kesseli}

Dr. med. et lic. phil., Chefredaktor

Das MedEd Symposium des Schweizerischen Instituts für ärztliche Weiter- und Fortbildung SIWF erlebte am 28. September in Bern bereits seine dritte Auflage. Den Verantwortlichen um SIWF-Präsident Werner Bauer gelang es erneut, ein dichtes, informatives und anregendes Programm zusammenzustellen.

In seiner Eröffnungsansprache rief der SIWF-Präsident dem Publikum mit einem Augenzwinkern in Erinnerung, dass der Begriff «Symposium» ursprünglich für geselliges Beisammensein, ja sogar «Gelage» steht. Auch wenn die Organisatoren ihr Symposium nicht in diesem Sinn verstanden haben möchten, soll es mehr sein als eine blosse Abfolge von Referaten. Vielmehr will es Denkanstösse geben und Diskussionen auslösen, die direkt oder indirekt zu einer Verbesserung der ärztlichen Weiterbildung beitragen. Die Horizonterweiterung durch den Blick ins Ausland gehört ebenfalls zum Programm.

\section{Veränderte Bedürfnisse von Patienten und Ärzteschaft}

Tradition haben am MedEd Symposium auch die Auftritte von Pascal Strupler, der zum dritten Mal ein Grusswort an die Anwesenden richtete. Die Frage, ob es eine solche Veranstaltung brauche, stelle sich mittlerweile nicht mehr, hielt der Direktor des Bundesamts für Gesundheit mit Blick auf die beiden ersten MedEd Symposien fest. Diskussionen entstünden nur dann, wenn ein Thema wichtig und in Bewegung sei. An Themen dieser Art habe an den bisherigen Symposien kein 


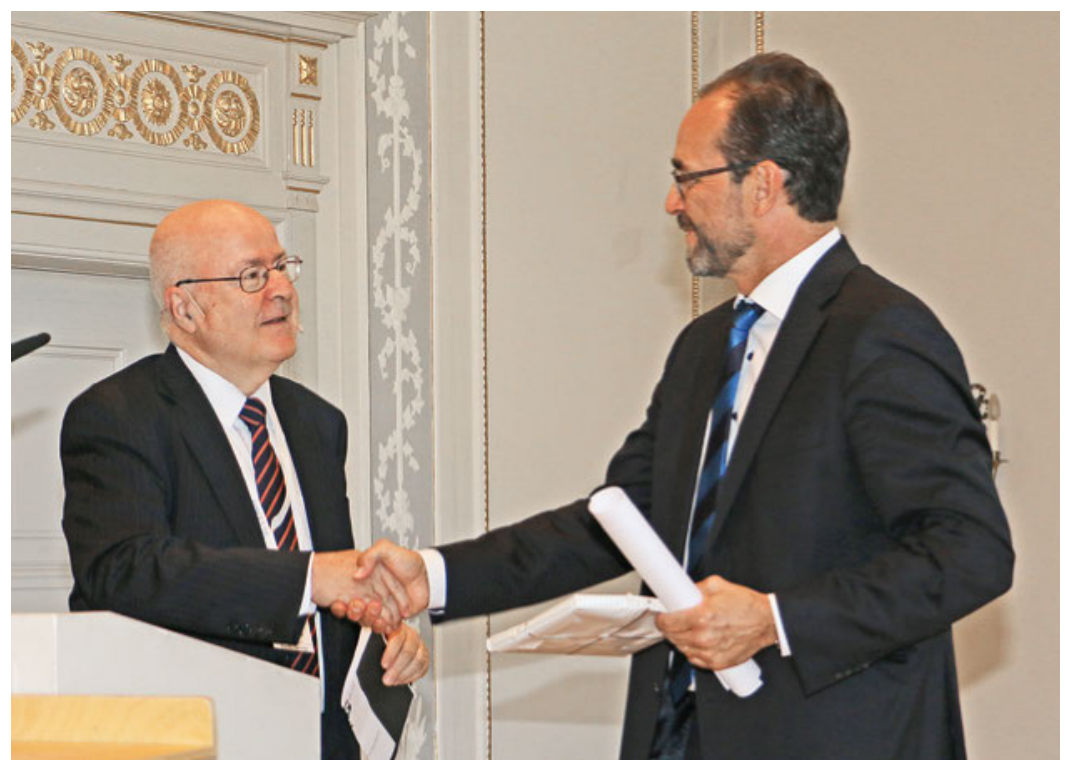

Werner Bauer (links) überreicht Pascal Strupler nach dessen Auftritt ein «Bhaltis» (kleines Geschenk)

Mangel geherrscht. Der BAG-Direktor erinnerte in diesem Zusammenhang an das provokative Referat von Johann Steurer am 2. Symposium [1]

Im Übrigen würdigte Strupler die Bedeutung der Ärzteschaft im Gesundheitswesen, deutete aber auch an, dass diese sich den Herausforderungen der Zeit stellen und zu Änderungen bereit sein müsse. Ärztinnen und Ärzte seien nach wie vor Grundpfeiler unserer Gesundheitsversorgung. Da sie weder vom Himmel fielen noch nach Belieben aus dem Ausland importiert werden könnten, «muss uns die ärztliche Bildung beschäftigen». Die Bedürfnisse der Patienten wie auch der Ärzteschaft änderten sich. Der Bundesrat versuche, auf die aktuellen und anstehenden Herausforderungen mit

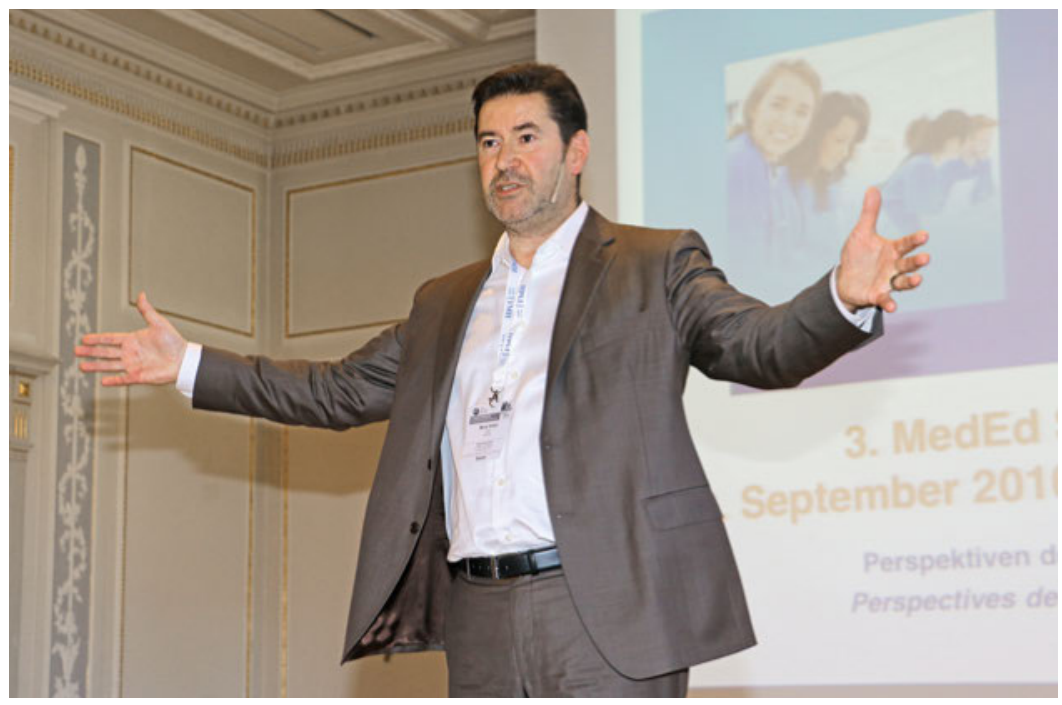

Selbstorganisation als Schlüsselkonzept: Marcel Altherr. der Strategie Gesundheit2020 wirkungsvolle Antworten zu geben.

Fragen der Steuerung, die mittlerweile eher als Koordination bezeichnet wird, sind auf verschiedenen Ebenen und "Plattformen" weiterhin ein Thema. Dazu gehört die Frage, wie viele Fachärztinnen und -ärzte es in den verschiedenen Disziplinen braucht, wie man auf die angestrebten Zahlen kommt und wie man die verschiedenen Spezialisten dorthin bringt, wo sie gebraucht werden. Ein weiteres Thema, das in der Diskussion und in der zukünftigen Gesundheitsversorgung eine wichtige Rolle spielt, ist die Interprofessionalität. Strupler liess zudem durchblicken, dass die Milderung oder Aufhebung des Vertragszwangs nach wie vor auf der Agenda verschiedener Stakeholder im Gesundheitswesen steht. Dass die Ärzteschaft dazu eine klare Haltung hat, ist ihm bewusst.

\section{SIWF als Mitgestalter}

Auch für Werner Bauer ist nicht alles eitel Sonnenschein im Gesundheitswesen und speziell im Bereich der ärztlichen Bildung, für die er zuständig ist. Die ärztliche Weiterbildung bezeichnete er im Titel seines Referats [2] gar als Sorgenkind. Als Sorgenkind mit Zukunft allerdings, so dass letztlich keine Zweifel daran aufkamen, dass er die anstehenden Aufgaben für lösbar hält. Das SIWF sieht er dabei keineswegs als blosse Verwaltungsinstanz, sondern in einer aktiven, gestaltenden Rolle. Seine Anliegen verdeutlichte er mit Bildern und Metaphern. In einer Zeit, da Methoden und Strukturen im Fluss seien, dürfe die Weiterbildung kein stehendes Gewässer bleiben, meinte er etwa. Netze seien nicht nur zum Fischen nützlich, und Gartenzäune, Mäuerchen und Silos dürften die Weiterbildungsdiskussionen nicht prägen und blockieren. Er machte unmissverständlich deutlich, dass er die Weiterbildung der kommenden Ärztegenerationen als undiskutable Verpflichtung des ärztlichen Berufsstandes sieht. Allerdings müssten für diese Aufgabe auch Zeit und finanzielle Mittel zur Verfügung gestellt werden. Ein Hinweis, der sich durchaus an die "Partner» im Gesundheitswesen richtete. Dagegen sei das Formulieren der $\mathrm{zu}$ erwerbenden Kompetenzen eine anspruchsvolle Aufgabe, der "wir» - also die Ärzteschaft - «uns stellen müssen».

\section{Das digitale Fieber greift um sich}

Dass Krankheiten ganze Institutionen erfassen können, legte der Mathematiker Marcel Altherr in seinem Referat «Das digitale Fieber» dar. «Alles, was digitalisiert werden kann, wird digitalisiert», formulierte er in 


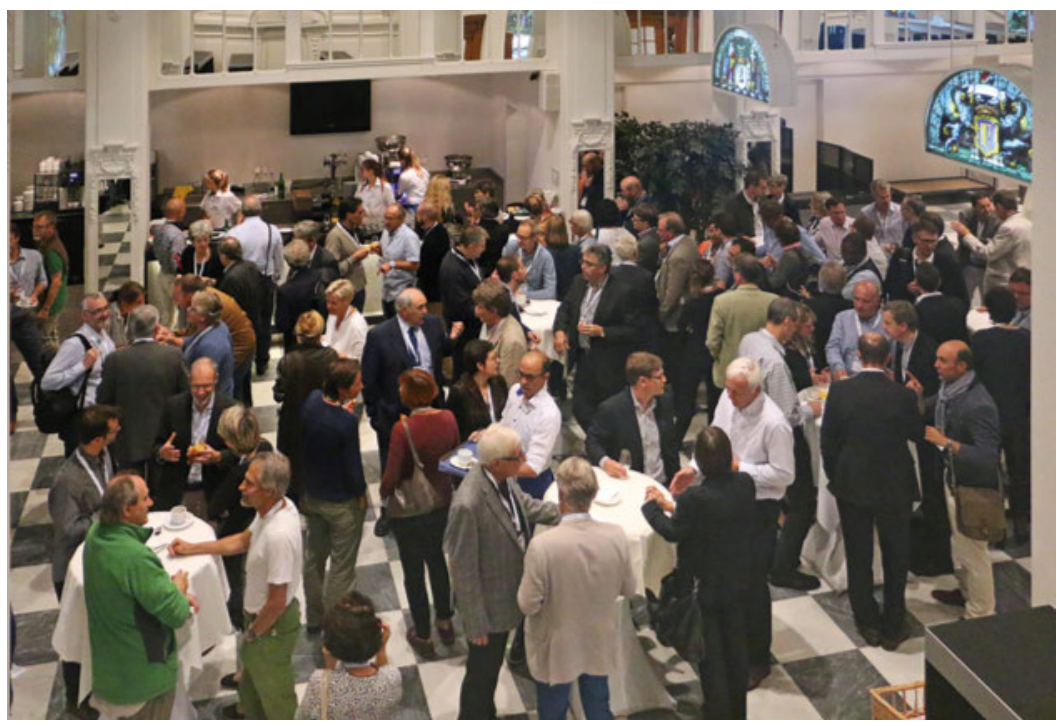

Auch ein Charakteristikum des MedEd Symposiums: angeregte Diskussionen in den Pausen.

Anlehnung an das bekannte Dürrenmatt-Zitat: "Alles Denkbare wird einmal gedacht.» Auch er brauchte starke Bilder, sprach von einem «digitalen Tsunami», der auch die medizinische Weiterbildung betreffen werde. Zwar betonte er, dass niemand vorhersagen könne, wie die neue Bildungslandschaft konkret aussehen werde. Klar ist für ihn aber, dass Organisationen - auch im Gesundheitswesen - in Zukunft ein neues «Betriebssystem» brauchen, wenn sie erfolgreich sein wollen. Die nach wie vor dominierende Organisationstheorie stamme aus dem 19. Jahrhundert und sei hoffnungslos veraltet. "Selbstorganisation" ist nach seiner Darstellung für erfolgreiche Organisationen in Zukunft das Schlüsselkonzept, und klassische Hierarchien werden durch «Verantwortungskreise» abgelöst.

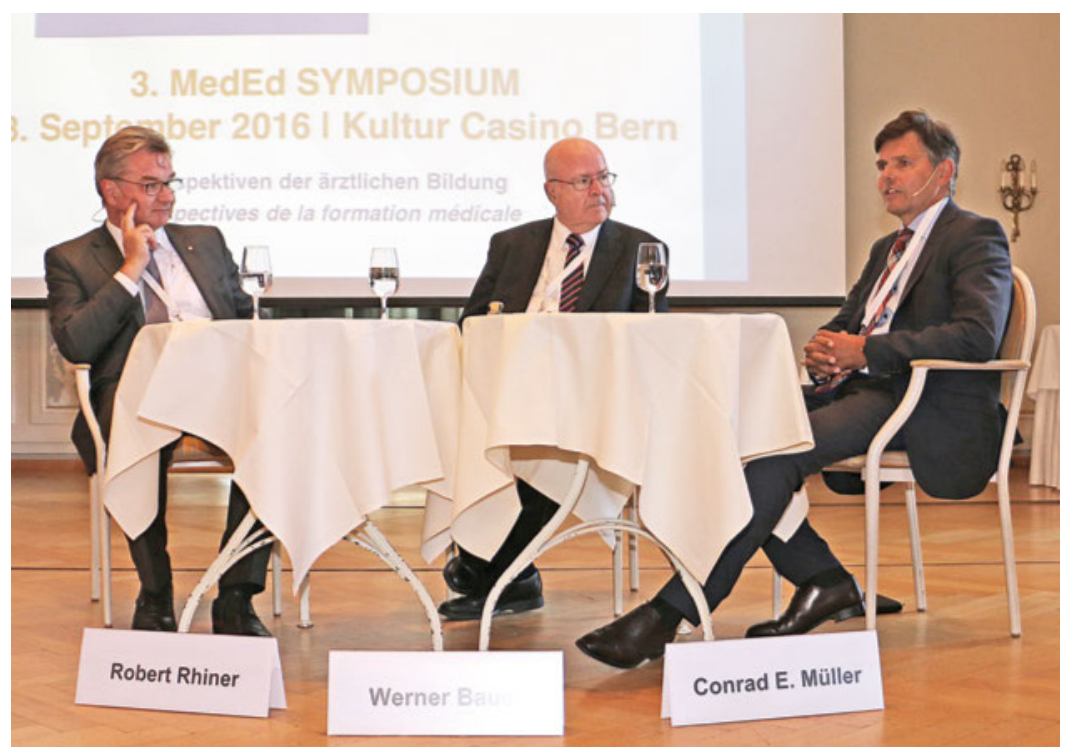

MedEd-Interview: Werner Bauer (Mitte) befragt Robert Rhiner (links) und Conrad E. Müller.
Diese Kombination führt zu «agilen Formen», die bereits in der Gegenwart von verschiedensten Unternehmen - auch in der Schweiz - sehr erfolgreich praktiziert werden. Interessanterweise behalten in diesen Modellen "gute alte Werte», darunter insbesondere das Vertrauen, einen hohen Stellenwert.

\section{Bekenntnis zur Weiterbildung unter erschwerten Bedingungen}

Vorwiegend um handfeste Fragen der Gegenwart ging es auch im MedEd-Interview, das Werner Bauer mit zwei Ärzten führte, die es mittlerweile in ihren Unternehmen an die Spitze geschafft haben: Robert Rhiner steht als CEO der Geschäftsleitung des Kantonsspitals Aarau vor, während Conrad E. Müller bei der Klinik Hirslanden Zürich das Amt des Direktors innehat. Müller betonte mehrfach, dass sich die Hirslanden Kliniken in der Weiterbildung engagieren wollen. Dass dabei auch Interessen im Zusammenhang mit der hochspezialisierten Medizin eine Rolle spielen, stellte er auf eine kritische Nachfrage von Werner Bauer nicht in Abrede, betonte aber, dass dies nicht der entscheidende Treiber sei. Kritische Stimmen aus dem Publikum wiesen etwa auf die bescheidene Anzahl Stellen hin, die Hirslanden im Bereich der Radiologie anbiete. Ihnen hielt Müller entgegen, der Aufbau entsprechender Strukturen brauche Zeit. Die Weichen seien aber richtig gestellt, unter anderem mit einem Sekretariat für Lehre. Robert Rhiner hielt für sein Spital fest, das Niveau des Teachings sei hoch, aber die fehlende Zeit und ein gewisser "Zwang zu teachen» könnten auf die Qualität drücken. Auch in dieser Runde zeigte sich, dass die nicht abschliessend gelöste Frage der Finanzierung der Weiterbildung von zentraler Bedeutung ist.

\section{Competency-Based Education hinterfragt}

Wie in vielen Lebensbereichen gibt es auch in der Medical Education Entwicklungen, von denen man nicht genau weiss, ob es sich dabei um echte Fortschritte oder eher um Modeströmungen handelt. Im Trend liegt zurzeit die Competency-oder Outcome-Based Education ( $C B E$ bzw. OBE), zu deren Instrumentarium beispielsweise die auch hierzulande bekannten Entrustable Professional Activities (EPAs) gehören. Der Kanadier Geoff Norman hinterfragte diese Konzepte und Methoden in seinem Referat kritisch. Dem Nuklearphysiker, Psychologen und Professor emeritus für klinische Epidemiologie und Biostatistik der McMaster University, Kanada, wird niemand vorwerfen, er wisse nicht, wovon er spreche. Ein zentraler Teil seiner Arbeit gilt der «Medical Education», deren Instrumente er 


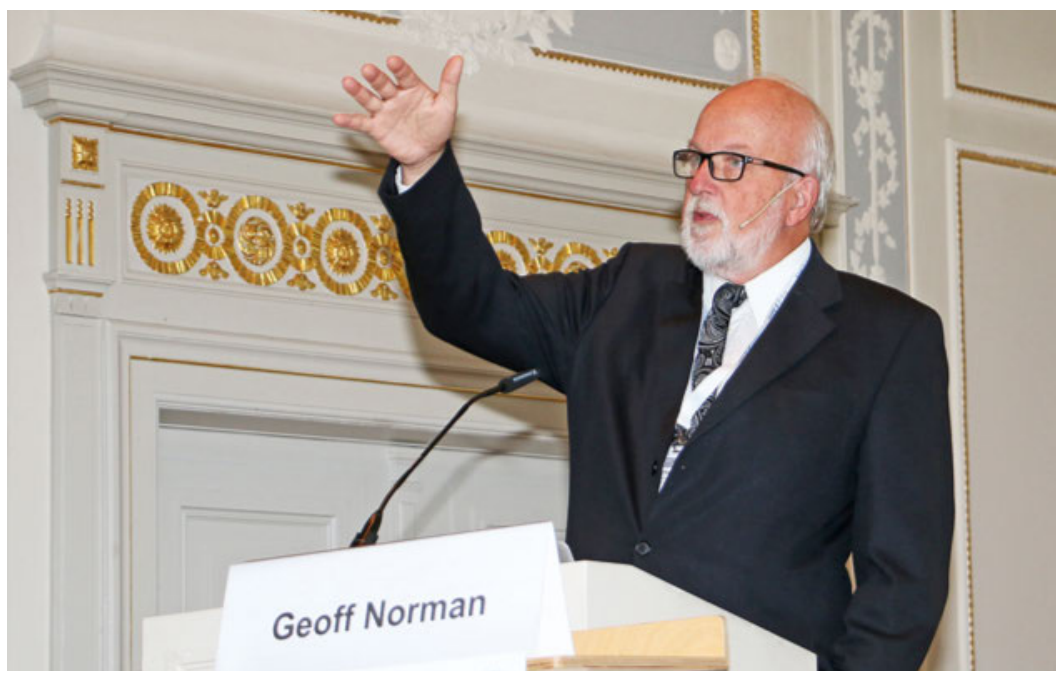

Kompetenzbasierte Ausbildungskonzepte kritisch hinterfragt: Geoff Norman.

wissenschaftlich erforschte und $\mathrm{zu}$ deren Weiterentwicklung er wichtige Beiträge leistete. Er machte sich mit zahlreichen Büchern und Artikeln zum Thema Lernen und Bildung einen Namen.

Norman stellte zunächst fest, dass die Grundidee einer kompetenzbasierten Medical Education durchaus überzeugt. In der Folge wies er akribisch nach, dass «der Teufel im Detail steckt» und die Versprechen der CBE nicht wie erwartet eingelöst werden können. Dazu gehört etwa die Vorstellung, dass eine kompetenzbasierte Ausbildung die Trainingszeit bis zum Erreichen eines bestimmten Kompetenzniveaus reduziert, dass diese Form der Ausbildung also effizienter ist als traditionelle Ausbildungsformen. Zudem ist nach seiner Erkenntnis eine verlässliche integrative Einstufung des Niveaus in Bezug auf alle verlangten Kompetenzen, das heisst eine Art globales Assessment, schlicht unmöglich. Dies gilt sogar schon für die einzelnen EPAs. Allerdings konnte er auch einen Lösungsansatz präsentieren, nämlich das Completed Clinical Evaluation
Report Rating (CCER). Dieses kombiniert eine Mikromit einer Makrostrategie. Mittels Checklisten werden diverse spezifische Aufgaben bewertet (Mikroebene) und diese Bewertungen dann zu globalen Assessments zusammengefasst (Makroebene). Werden solche Bewertungen genügend häufig durchgeführt - in einer gezeigten Studie war es täglich der Fall -, führt diese Methode offenbar zu klaren und gut dokumentierbaren Fortschritten der Auszubildenden bei einer hohen «Interrater reliability». Es wird sich zeigen, ob das CCER sich durchsetzen und allenfalls auch in unseren Breiten Schule machen wird.

\section{Wird es besser ohne Zeit und Geld?}

Der Nachmittagsteil wurde durch drei parallel geführte Seminarien [2] eingeleitet, in denen folgende Themen behandelt und intensiv diskutiert wurden:

- Und wenn den Bildungsexperten die technologische Entwicklung davonrennt?

- Weiterbildung der Zukunft: strukturierte Curricula in Netzwerken?

- Diagnostic error mechanisms and prevention: an utopia?

Danach ging Martin Krause einer etwas sarkastisch formulierten Frage nach. «Regulierte Weiterbildung ohne Zeit und Geld: Wird's wirklich besser?», lautete der Titel seines Referats. Die sehr humorvoll vorgetragenen und mit witzigen Anekdoten veranschaulichten Erkenntnisse und Erfahrungen des internistischen Chefarztes des Spitals Münsterlingen provozierten immer wieder Gelächter, das zweifelsohne als zustimmend interpre-

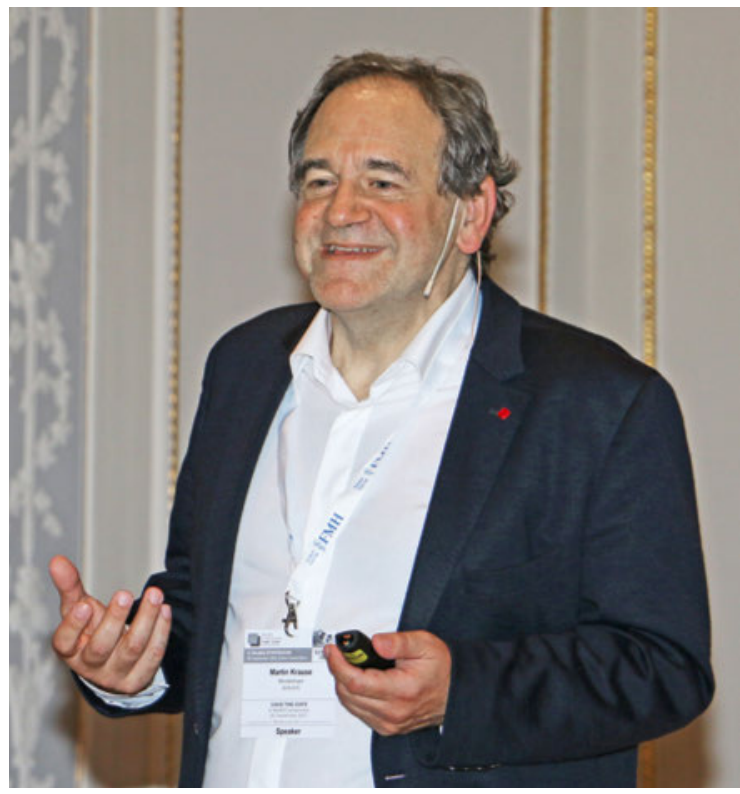

«Nah am Patienten»: Martin Krause.

\section{SIWF-Award 2016: die Ausgezeichneten}

Im Rahmen des 3. MedEd-Symposiums wurden auch die Trägerinnen und Träger des SIWFAwards 2016 für besonderes Engagement in der Weiterbildung bekanntgegeben. Diese Auszeichnung wird aufgrund der Nomination durch ehemalige Assistenzärztinnen und -ärzte vergeben. Folgende Weiterbildner erhielten in diesem Jahr eine Auszeichnung:

Prof. Dr. med. Romaine Arlettaz, Zürich; Dr. med. Stefan Arndt, Münsterlingen; Dr. med. Esther Bächli, Uster; Dr. med. Arménio Barata, Biel; Dr. med. Patrick Bodenmann, Lausanne; Dr. med. Charles Dvorak, Vallorbe; Prof. Dr. med. Dominique Erni, Bern; Dr. med. Bianka Freiwald, Baden; Prof. Dr. med. Emanuel Gautier, Freiburg; Prof. Dr. med. Andreas Günthert, Luzern; Dr. med. Samuel Henz, St. Gallen/Rorschach; Dr. med. Tobias Hübner, Münsterlingen; Dr. med. Orpheus Kolokythas, Winterthur; Silvia Lambiel, Genf; Dr. med. Adrian Marty, Zürich; Dr. med. Stefan Mariacher-Gehler, Zollikerberg; Dr. med. Friederike Meyer zu Bentrup, Visp; Dr. med. Marco Negri, Frutigen; Dr. med. habil. Sebastian Olbrich, Zürich; Prof. Dr. med. Nicolas Regamey, Luzern; Dr. med. lanina Scheer, Zürich; Dr. med. Renato Scognamiglio, Freiburg; PD Dr. med. Martin Siegemund, Basel; Dr. med. Nils Siegenthaler, Genf; Dr. med. Daniel Studer, Basel. 


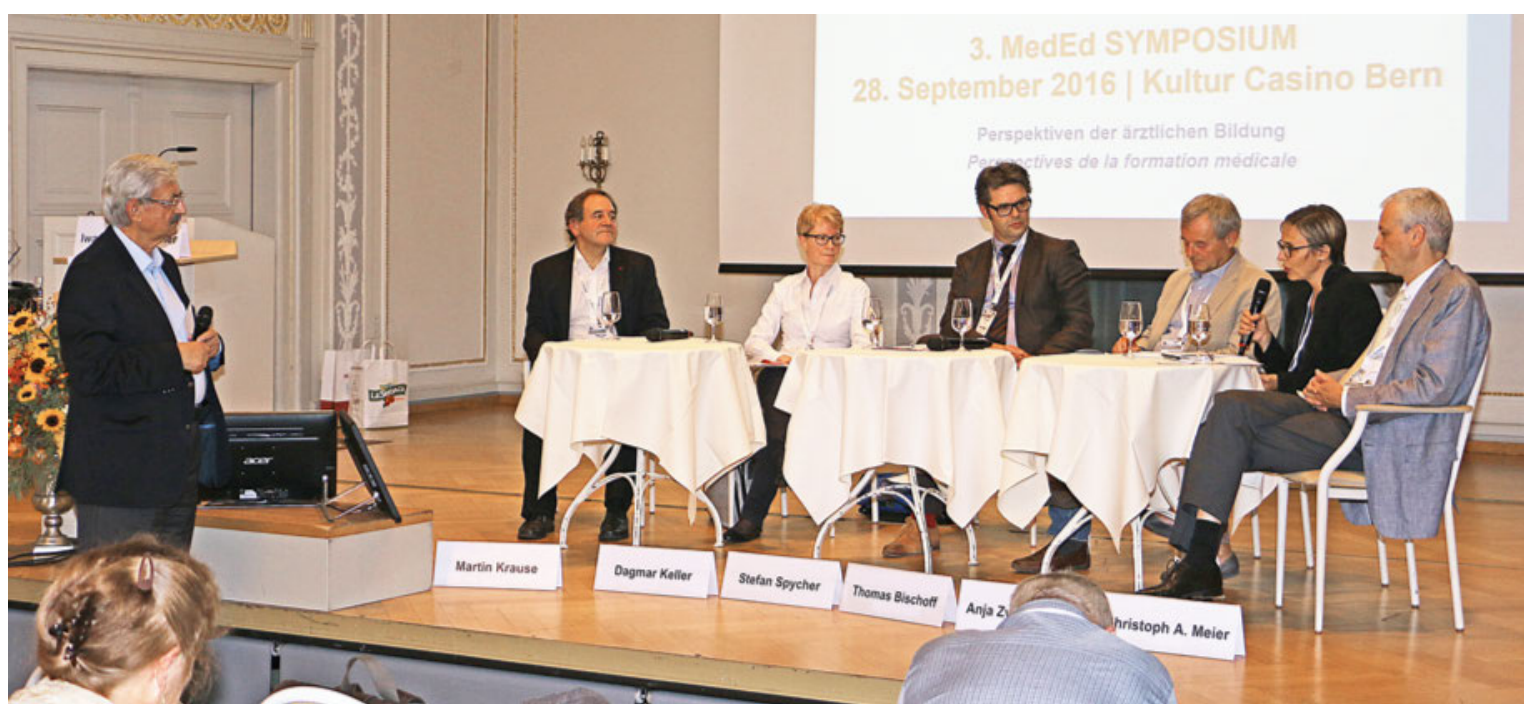

Diskutierten engagiert (von links): Iwan Rickenbacher (Diskussionsleitung), Martin Krause, Dagmar Keller, Stefan Spycher, Thomas Bischoff, Anja Zyska, Christoph A. Meier.

tiert werden durfte. Krause plädierte für eine patientennahe Medizin, in der die klinische Untersuchung und die Zuwendung zum Patienten nach wie vor einen hohen Stellenwert haben sollen. Er zeigte sich überzeugt, dass dies der Qualität der Weiterbildung zugutekomme und auch den eigentlichen Wünschen der Assistenzärztinnen und -ärzte entspreche, die «gern nahe am Patienten" seien. Dem SIWF attestierte er gute Arbeit. Der sanfte Druck von oben habe dazu beigetragen, dass die Weiterbildung in den letzten Jahren besser geworden sei.

Auf dem abschliessenden Podium wurden von Moderator Iwan Rickenbacher sehr konkrete Fragen der Weiterbildung angesprochen. Die Podiumsgäste [3] diskutierten engagiert über Qualität, Dauer und Finanzierung der Weiterbildung, aber auch über die Entwicklung der Arbeitsbedingungen in Spital und Praxis, die sich wandelnden Bedürfnisse der Patienten und der jungen Ärztegeneration, die Herausforderungen durch den Strukturwandel und die Chancen und Risiken des

\section{Projektförderung SIWF 2016}

Die SIWF-Projektförderung ist ein Instrument zur Verbesserung der ärztlichen Weiterbildung. Folgende Projekte wurden 2016 unterstützt:

- Une application pour améliorer l'apprentissage de l'auscultation cardiopulmonaire par les médecins-assistants; Dr méd. David Gachoud, MEd; CHUV

- Einführung des Multi-Source Feedback in der ärztlichen Aus- und Weiterbildung; Dr. med. Barbara Fiedel und Dr. med. Severin Meili; Kantonsspital Winterthur

- Entwicklung und Implementierung einer generischen elektronischen Plattform für Workplace Based Assessments (e-WPBA); Dr. med. Gianmarco Balestra und Dr. med. Matthias Hepprich; Universitätsspital Basel

Kurzbeschreibungen der unterstützten Projekte finden Sie in der Online-Ausgabe unter www.saez.ch $\rightarrow$ Archiv $\rightarrow$ Zeitschriftenarchiv $\rightarrow$ 2016 $\rightarrow$ Ausgabe 46 . technologischen Fortschritts. In vielen Punkten war sich die Runde einig. So wurde es als wünschenswert erachtet, die Weiterbildungsdauer wenn möglich zu verkürzen. Der Stellenwert des Teachings und die Vorbildfunktion der Weiterbildenden wurden unterstrichen, und das Problem des Hausärztemangels - und wirksame Gegenmassnahmen - wurde als vordringlich eingestuft. Empathie und Patientennähe wurden auch im Zeitalter der High-Tech-Medizin hoch bewertet. «Wir sollten uns nicht mit High-Tech profilieren», hielt Christoph A. Meier fest, «sondern mit vernünftiger, patientenorientierter Medizin.»

Einigkeit dürfte bei allen Symposiumsteilnehmern auch darüber geherrscht haben, dass der Diskussionsstoff im Bereich der Medical Education nicht ausgehen wird. An möglichen Themen wird es also auch dem 4. MedEd Symposium nicht fehlen, das am 20. September 2017 stattfindet.

\section{Literatur}

1 Eine Zusammenfassung findet sich in: Kesseli B. Zukünftigen Herausforderungen wirkungsvoll begegnen. Schweiz Ärztezeitung. 2015;96(45):1638-41.

2 Die Präsentationen zu den Hauptreferaten und Seminarien sind unter www.fmh.ch/bildung-siwf/themen/meded-symposium.html zugänglich.

3 Auf dem Podium diskutierten: Prof. Dr. med. Thomas Bischoff, Vice-Directeur École de formation postgraduée (EFPG) CHUV, Lausanne; Prof. Dr. med. Dagmar Keller, Direktorin, Institut für Notfallmedizin, UniversitätsSpital Zürich; Prof. Dr. med. Martin Krause, Chefarzt Medizinische Klinik, Kantonsspital Münsterlingen; Prof. Dr. med. Christoph A. Meier, Ärztlicher Direktor, Universitätsspital Basel; Dr. med. Anja Zyska, Vizepräsidentin Verband Schweizerischer Assistenz- und Oberärztinnen und -ärzte VSAO Dr. Stefan Spycher, Vizedirektor, Leiter Direktion Gesundheitspolitik, Bundesamt für Gesundheit (BAG). 


\section{SIWF ISFM}

\section{Kardiopulmonale Auskultation: besserer Lernprozess für Assistenzärztinnen und Assistenzärzte}

Seit der Erfindung des Stethoskops durch Laennec im Jahr 1816 ist die Auskultation eine zentrale Massnahme der klinischen Untersuchung. 200 Jahre danach sind dank dem technologischen Fortschritt neue leistungsfähige Diagnoseinstrumente wie der Ultraschall verfügbar, mit dem sich eine Untersuchung am Krankenbett einfach durchführen lässt. Trotzdem hat die Auskultation nach wie vor eine wichtige Funktion, zumal auch sie durch den technischen Fortschritt verbessert wurde. Damit ist die Auskultation eine sinnvolle Ergänzung der übrigen verfügbaren Diagnoseinstrumente.

Die kardiopulmonale Auskultation beruht selbstverständlich darauf, dass der Arzt in der Lage ist, die Herz- und Lungentöne richtig zu erkennen. Aus mehreren Studien geht jedoch hervor, dass die diesbezüglichen Kompetenzen der Ärztinnen und Ärzte und insbesondere der Assistenzärztinnen und Assistenzärzte teilweise unzureichend sind.

Bei diesem Projekt geht es darum, die Kompetenzen der Assistenzärztinnen und Assistenzärzte im Bereich der kardiopulmonalen Auskultation zu verbessern. Dazu soll der technologische Fortschritt genutzt werden, der für die Weiterentwicklung des Stethoskops ausschlaggebend war. Das elektronische Stethoskop ermöglicht nicht nur eine optimierte Übertragung der Herz- und Lungentöne, sondern auch den gleichzeitigen Zugang zum jeweiligen Tonspektrogramm mit Hilfe eines Smartphones. Damit steht dem Arzt eine Kombination von akustischen und visuellen Signalen zur Verfügung, dank denen er die Herz- und Lungentöne besser erkennen kann.

Dieses Projekt hat den Zweck, eine Smartphone-Applikation zu entwickeln. Dank dieser App werden die Assistenzärztinnen und Assistenzärzte Zugang zu den akustischen und visuellen Signalen haben, die als Streaming von einem elektronischen Stethoskop übertragen werden, das von einem beaufsichtigenden Arzt im Rahmen der Ausbildung am Patientenbett oder während einer Arztvisite benutzt wird.

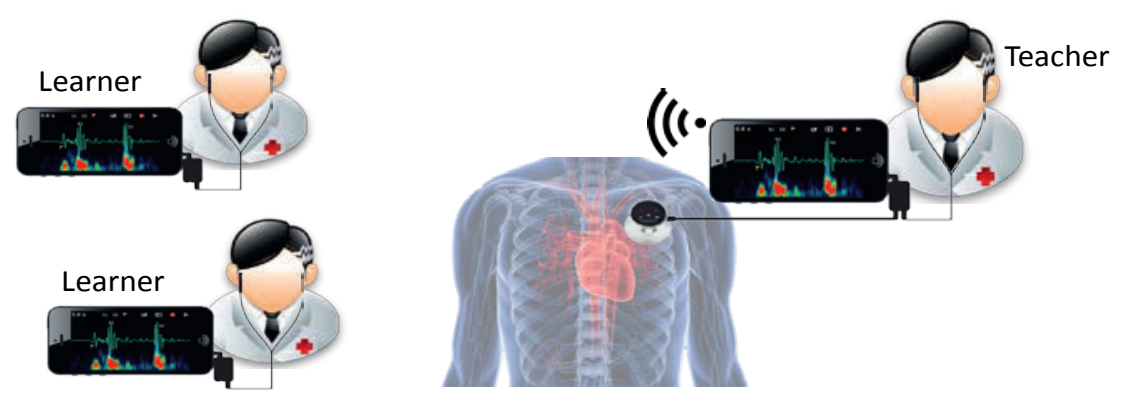

Das Projekt entstand aus der Zusammenarbeit zwischen der Abteilung für Innere Medizin des CHUV in Lausanne (Dr. David Gachoud, Dr. Matteo Monti und Prof. Gérard Waeber), der pädagogischen Abteilung der Fakultät für Biologie und Medizin der Universität Lausanne (Dr. Raphaël Bonvin) und dem Unternehmen Smartcardia (ein Spin-Off der Eidgenössischen Technischen Hochschule Lausanne; Dr. Srinivasan Murali und Dr. Francisco Rincon). 


\section{SIWF \\ ISFM}

\section{Einführung des Multisource Feedback (MSF) in der ärztlichen Aus- und Weiterbildung}

Neben einer steten Qualifikation der fachlichen Fähigkeiten ist im modernen medizinischen Curriculum auch ein strukturiertes Feedback über die kommunikativen und sozialen Fähigkeiten der Mitarbeiter und Führungskräfte mit Kontrolle von Gelerntem und Formulierung von neuen Zielen essentiell.

Mit der Einführung des Multisource Feedback (MSF), welches in vielen anderen Berufsbranchen und Führungsetagen seit Jahrzehnten etabliert ist, kann diesen Herausforderungen adäquat begegnet und die Entwicklung einer Arbeitskultur der Zukunft vorangetrieben werden. Im englischen Gesundheitssystem wird das MSF durch das Royal College of Physicians seit vielen Jahren erfolgreich angewandt.

In Phase 1 wird das MSF bei den Assistenzärzten (AA) durchgeführt. Jeder AA wählt 12-15 Mitarbeiter verschiedener Rollen (AA, Pflege, Kaderarzt) in seinem Umfeld aus, welche anonym anhand eines Fragebogens eine Einschätzung über seine zwischenmenschlichen Kompetenzen abgeben. Zusätzlich führt der AA eine Selbsteinschätzung durch. Die Synthese dieser Selbst- und Fremd-Einschätzungen wird mit einem Tutor (im Feedback speziell geschulter Kaderarzt) besprochen, um individuelle Potentiale zu erkennen und neue Ziele zu setzen. Dieses Feedback wird regelmässig wiederholt.

Das MSF muss effizient durchführbar sein und klare, verwertbare Ergebnisse liefern. Die Befragung erfolgt online anonym und wird automatisch ausgewertet. Für die professionelle Umsetzung dieses Teils arbeiten wir mit einer auf diesem Gebiet sehr erfahrenen Firma zusammen.

In der zweiten Phase wird das MSF auf die Kadermitarbeiter ausgeweitet. Hierbei bedarf es Anpassungen im Prozess. Für eine akzeptierbare und gewinnbringende Besprechung der Feedbackergebnisse mit dem Kader werden externe Coachs eingesetzt.

Eine Nutzung für das gesamte Kantonsspital Winterthur (KSW) und andere Spitäler ist in einer 3. Phase angedacht. 


\title{
Entwicklung und Implementierung einer generischen elektronischen Plattform für Work-place Based Assessments - eWPBA
}

\author{
Dr. med. Matthias Hepprich ${ }^{1}$, Dr. med. Gianmarco M. Balestra ${ }^{2,3}$
}

Arbeitsplatz-basierte Assessments (WBPA) wie Mini-CEX und DOPS wurden in der Schweiz zur Qualitätssteigerung der ärztlichen Weiterbildung eingeführt und dienen als Basis für Supervision und Feedback von Studierenden und Assistenzärzten. Ziel ist die Erlangung ärztlicher Kompetenzen zu optimieren. Hierfür ist das qualifizierte Feedback von Klinikern entscheidend. Es gibt allerdings zahlreiche Limitationen, die einen effektiven und effizienten Einsatz der WBPA erschweren.

Wesentliche Kritikpunkte der WBPA sind der zeitliche Aufwand, die Bindung personeller Ressourcen, die Notwendigkeit, Formulare in Papierform zu verwenden sowie geringe Vertrautheit mit den Bewertungswerkzeugen respektive -kriterien und mangelnde Anleitung, wie gutes Feedback-Geben aussehen soll.

Während Zeit- und Personalaufwand format-inhärent sind, können die übrigen Aspekte modifiziert werden. Wir entwickeln eine Applikation (App), die eine einfach zu nutzende, strukturierte Beurteilung von WBPA ermöglicht. Mit der von uns geplanten generischen, elektronischen Plattform soll eine qualitativ verbesserte und zeiteffiziente Durchführung von WBPA möglich sein. Ausserdem stehen die WPBA-Daten langfristig und detailreicher für den Lernprozess und die Leistungsauswertung zur Verfügung. Die Alpha-Version ist auf iPhone ${ }^{\circledR}$ nutzbar und soll zukünftig auch auf anderen mobilen Endgeräten verwendbar sein. Wir erwarten eine verbesserte Anwendbarkeit und Akzeptanz im klinischen Alltag durch Vereinfachung des Beurteilungsprozesses, geringeren administrativen Aufwand und Anleitung zum differenzierten Feedback. Nach der Projektphase wird die App am Universitätsspital Basel implementiert und evaluiert. Das App-Design und die zugrundeliegende Softwarearchitektur zielen darauf ab, breit in der Aus- und Weiterbildung auch an anderen Schweizer Spitälern verwendet zu werden. Schnittstellen zu weiterer Software (z.B. FMH e-Logbuch) werden mitberücksichtigt und bieten Integrationsspielraum.

\footnotetext{
${ }^{1}$ Klinik für Endokrinologie, Diabetologie und Metabolismus, Universitätsspital Basel, Petersgraben 4, 4031 Basel

${ }^{2}$ Medizinische Intensivstation, Universitätsspital Basel, Petersgraben 4, 4031 Basel

${ }^{3}$ Dekanat Medizinische Fakultät, Universität Basel, Klingelbergstrasse 61, 4056 Basel
} 